\title{
COMPREHENSIVE QUALITY ASSESSMENT METHOD FOR THE TRADITIONAL CHINESE MEDICINE COMPLEX SYSTEM ON THE BASIS OF HOLISM
}

\section{() Luo Guo-An, Liang Qiong-lin, Wang Yi-ming}

\author{
Tsinghua University, P. R. China
}

The presentation summarized the recent development and progress of methodologies, techniques and regulations of China, focused on the field of comprehensive quality assessment method for the TCM complex system. Five main aspects were addressed: 1) Proposal of the comprehensive quality assessment system for Traditional Chinese Medicine (TCM). A comprehensive quality assessment method based on the combination of macrocosmic characterization and microscopic feature for the TCM complex systems were introduced. 2) Introduction of TCM fingerprint. The use of chromatographic fingerprinting combined with quantitation of multi-indicative components for the quality control of TCM and botanicals were described here. Many new fingerprinting techniques, such as multi-dimensional fingerprint, have been applied for the overall characterization of chemical composition of TCM complex systems, such as Qingkailing injection, ginseng products, and so on. The development of the software for information processing of TCM fingerprint can be applied for not only the similarity evaluation but also the classification, recognition and identification of the fingerprint based on correlation analysis and discriminant analysis. 3) Introduction of Quantitative Analysis of Multi-Components by Single-marker. Since the importance of multi-component determination and the difficulty in acquiring the reference standards, a new strategy named as Quantitative Analysis of Multi-Components by Single-marker (QAMS) have been proposed and applied in 2010 Chinese pharmacopeia. We took ginseng products, Fructus of Gardenia jasminoides Ellis and herba epimedii as example here to be discussed the feasibility and universality of QAMS. 4) Bio-Response fingerprint for assessment of efficacy and safety of TCM. How to charecterize and express the quality of TCM complex system corresponding to the efficacy and safety is a critical but difficult target. The roadmap of gene chip technique applied in the quality evaluation of TCM proposed here. Some examples, such as venenum bufonis, were reported here.

\section{GENERAL TOXICITY STUDY OF THE NEW MEDICATION BASED ON THE SCHISANDRA CHINENSIS SEEDS}

() Makarenko I. E., Avdeeva O. I., Makarova M. N., Pozharitskaya O. N., Kryshen K. L.

St-Petersburg Institute of Pharmacy, Russia

General toxicity study (acute and chronic) is the part of preclinical program for safety evaluation of new pharmaceuticals. In the acute toxicity study toxic, maximum tolerable and lethal doses for rats and mice were determined. The aim of the chronic toxicity study was to determine the safety profile of new medicine based on the oil extract of the Schisandra Chinensis seeds. The experiments were carried out according to Russian legislation of Ministry of Health and Social Development and international guidelines in the preclinical safety evaluation and Good Laboratory Practice (GLP) standarts and approved by local ethical commission. For acute toxicity study the tested drug was administered oral and intraperitoneal in five doses 500, 2000, 5000, 10000 and $15000 \mathrm{mg} / \mathrm{kg}$ (of oil extract) to outbred female and male rats and mice in the same doses for each type of animals. Each group consisted of 5 animals of both sexes. In chronic experiment the tested medicine was administered intraperitoneal during 90 days in doses 50,500 and $1000 \mathrm{mg} / \mathrm{kg}$ (of oil extract) to outbred female and male rats ( 10 animals per group). In acute experiment LD50 was not determined due to the absence of experimental animals lethality. Because the maximum dose for oral treatment was $15000 \mathrm{mg} / \mathrm{kg}$ the tested pharmaceutical can be attributed to Class VI relatively harmless compounds according to the classification of Hodge and Sterner (1). In chronic experiments on the laboratory rats and mice there was no any toxic effects after 90 days of treatment for tested drug in all doses.

Reference: (1) Hodge H.C. and Sterner L. H. Tabulation of toxicity classes. Am. Industr. Hyg. Ass. Quart., 1943, 10 (4): 93. 